\title{
A semigroup approach to wreath-product extensions of Solomon's descent algebras
}

\author{
Samuel K. Hsiao \\ Mathematics Program \\ Bard College \\ Annandale-on-Hudson, NY, 12504 \\ hsiao@bard.edu
}

Submitted: Aug 15, 2008; Accepted: Jan 27, 2009; Published: Feb 4, 2009

Mathematics Subject Classification: 05E99; 16S34; 20M25

\begin{abstract}
There is a well-known combinatorial model, based on ordered set partitions, of the semigroup of faces of the braid arrangement. We generalize this model to obtain a semigroup $\mathcal{F}_{n}^{G}$ associated with $G$ ? $S_{n}$, the wreath product of the symmetric group $S_{n}$ with an arbitrary group $G$. Techniques of Bidigare and Brown are adapted to construct an anti-homomorphism from the $S_{n}$-invariant subalgebra of the semigroup algebra of $\mathcal{F}_{n}^{G}$ into the group algebra of $G$ < $S_{n}$. The colored descent algebras of Mantaci and Reutenauer are obtained as homomorphic images when $G$ is abelian.
\end{abstract}

\section{Introduction}

A celebrated result of Solomon [27] reveals the existence of an intriguing subalgebra, known as the descent algebra, inside the group algebra of any finite Coxeter group. In the case of the symmetric group, the descent algebra has a particularly simple combinatorial interpretation in terms of descent sets of permutations. This interpretation is an important ingredient in numerous extensions, applications, and further investigations $[13,5,12,18,22]$. A fitting example, one that is central to this paper, is Mantaci and Reutenauer's construction of "colored" descent algebras [18] via wreath-product extensions of the symmetric group. Their work highlights the vibrant interest in developing colored versions of combinatorial tools associated with the symmetric group. Along these lines, a significant development is Baumann and Hohlweg's [4] far-reaching descent theory for wreath products, in which the functorial nature of the colored constructions are brought to light. Continuing in this vain, Bergeron and Hohlweg [6] provide a unifiying generalization of a number of colored constructions in the literature and discover new colored algebraic structures using their theory. Also part of this circle of ideas is Novelli and Thibon's [20,21] generalization of free quasisymmetric functions to the context of 
colored permutations, as well as Petersen and this author's [16] use of colored posets to study the Hopf algebraic structure of Poirier's colored quasisymmetric functions [24]. All of these works in some sense expand on the theme of colored descent algebras, and are thus part of a story began by Mantaci and Reutenauer.

This paper completes another part of the colored story by offering a wreath-product version of a semigroup theoretic approach to understanding to the descent algebra, an approach that goes back to the work of Tits [30, 31]. In his appendix to Solomon's paper $[27,31]$, Tits uses a semigroup structure on the faces of a Coxeter complex (which he states in terms of projection maps [30]) to prove and give geometric interpretations of Solomon's results. Building on Tits's ideas, Bidigare [7] explains how the descent algebra of the symmetric group $S_{n}$ can be recovered from the invariants of an $S_{n}$-action on the semigroup of faces of the braid arrangement, or equivalently, faces of the Coxeter complex of $S_{n}$. We will denote this semigroup by $\mathcal{F}_{n}$. Our goal is to generalize Bidigare's approach to $G<S_{n}$, the wreath product of $S_{n}$ with an arbitrary finite group $G$.

We learned of Bigidare's (unpublished) result through Brown's paper [9, Theorem 7], in which a geometric version of Bidigare's proof is given. While our proof is purely algebraic, it has Brown's argument at its core. The first step in our approach is to take a group $G$ and define a semigroup $\mathcal{F}_{n}^{G}$, which can be viewed as a wreath-product version of the face semigroup $\mathcal{F}_{n}$. Our semigroup is defined in terms of ordered set partitions of $\{1,2, \ldots, n\}$ decorated with elements of $G$, generalizing the combinatorial definition of $\mathcal{F}_{n}$. Unlike the face semigroup of the braid arrangement (or of a hyperplane arrangement in general), elements of $\mathcal{F}_{n}^{G}$ are not necessarily idempotent. Instead, they satisfy the identities

$$
x^{|G|+1}=x \quad \text { and } \quad x y x^{|G|}=x y
$$

for all $x, y \in \mathcal{F}_{n}^{G}$. When $|G|=1$ these identities define left regular bands. If $|G|$ is an arbitrary positive integer, a finite semigroup that satisfies (1) is an example of a left regular band of groups. Left regular bands of groups belong to the class of completely regular semigroups. See for example [23].

The next step in our approach is to introduce an $S_{n}$-action on the semigroup algebra $\mathbb{Z} \mathcal{F}_{n}^{G}$, for which the invariant subalgebra $\left(\mathbb{Z} \mathcal{F}_{n}^{G}\right)^{S_{n}}$ has a basis $\left(\sigma_{\alpha}\right)$ indexed by $G$-compositions (which generalize the notion of descent set). The group algebra $\mathbb{Z}\left[G<S_{n}\right]$ also contains a $\mathbb{Z}$-submodule, defined analogously to Solomon's descent algebra, having a natural basis $\left(X_{\alpha}\right)$ indexed by $G$-compositions.

Our main result, a wreath-product extension of Bidigare's theorem in the case of the symmetric group, is as follows:

Theorem 1. The $\mathbb{Z}$-module map $f:\left(\mathbb{Z}_{n}^{G}\right)^{S_{n}} \rightarrow \mathbb{Z}\left[G \backslash S_{n}\right]$ given by $f\left(\sigma_{\alpha}\right)=X_{\alpha}$ is an injective anti-homomorphism of algebras.

It follows that the image of $f$ is a subalgebra of $\mathbb{Z}\left[G<S_{n}\right]$. For abelian groups $G$ these subalgebras turn out to be the generalized descent algebras introduced by Mantaci and Reutenauer [18]. For arbitrary groups $G$ these algebras appear in the works of Novelli and Thibon [20] and Baumann and Hohlweg [4]. Also, see [2, 3, 15, 8] for works that make connections to the important special case $G=\mathbb{Z} / 2 \mathbb{Z}$. 
Our approach, in addition to providing an elementary and concise route to understanding Mantaci and Reutenauer's colored descent algebras, offers a combinatorial framework in which to explore or apply wreath-product versions of results on left regular bands. As a case in point, in a recent paper Margolis and Steinberg [19] develop a homology theory for the algebra of a regular semigroup, allowing them to compute the quiver of a left regular band of groups (extending Saliola's result for left regular bands [25, 26]), and as an application they explicitly describe the quiver of the semigroup algebra $\mathbb{Z} \mathcal{F}_{n}^{G}$. Another direction in which to look for colored versions is Brown's theory of Markov chains on left regular bands $[9,10]$. Steinberg $[28,29]$ has already generalized the spectral computation in [9] to semigroups with basic algebras, which include the semigroup $\mathcal{F}_{n}^{G}$. A related area that remains to be fully investigated is a wreath-product extension of the work of Hersh and this author [14] on interpreting endomorphisms of quasisymmetric functions as Markov chains; much of the machinery is already in place for such an investigation $[6,16]$. Let us also mention the monograph by Aguiar and Mahajan [1], who study the relationships between various combinatorial Hopf algebras through the lens of Coxeter groups and their associated face semigroups. Actually they work at the level of left regular bands. The functorial nature of many of the colored constructions of Baumann and Hohlweg [4] and Bergeron and Hohlweg [6] gives hope of extending the tools in [1] via wreath products to a broader class of semigroups, which would certainly include $\mathcal{F}_{n}^{G}$ but could perhaps be extended to left regular bands of groups or beyond.

Throughout this paper we assume that $G$ is a finite group and denote its identity element by $1_{G}$. However, it should be noted that the definitions of our main objects of study, namely $\mathcal{F}_{n}^{G},\left(\mathcal{F}_{n}^{G}\right)^{S_{n}}, G \prec S_{n}$, and $\mathcal{D}\left(G \succ S_{n}\right)$, still make sense when $G$ is an infinite group, or even just a semigroup with identity, that is, a monoid. For such $G$, Theorem 1 still holds, but Formulas (1) might fail.

\section{Preliminaries}

\subsection{The semigroup of ordered $G$-partitions}

Fix a positive integer $n$, and let $[n]=\{1,2, \ldots, n\}$. An ordered partition (also called a block partition) of $[n]$ is a tuple $\left(B_{1}, \ldots, B_{k}\right)$ of nonempty pairwise disjoint sets whose union is $[n]$.

An ordered $G$-partition of $[n]$ is a tuple $\left(\left(B_{1}, g_{1}\right), \ldots,\left(B_{k}, g_{k}\right)\right)$ such that $\left(B_{1}, \ldots, B_{k}\right)$ is an ordered partition of $[n]$ and $g_{i} \in G$ for all $i \in[k]$.

Let $\mathcal{F}_{n}^{G}$ denote the set of ordered $G$-partitions of $[n]$. Define multiplication in $\mathcal{F}_{n}^{G}$ by

$$
\left(\left(B_{1}, g_{1}\right), \ldots,\left(B_{k}, g_{k}\right)\right)\left(\left(C_{1}, h_{1}\right), \ldots,\left(C_{\ell}, h_{\ell}\right)\right)=\begin{gathered}
\left(\left(B_{1} \cap C_{1}, h_{1} g_{1}\right), \ldots,\left(B_{1} \cap C_{\ell}, h_{\ell} g_{1}\right),\right. \\
\left(B_{2} \cap C_{1}, h_{1} g_{2}\right), \ldots,\left(B_{2} \cap C_{\ell}, h_{\ell} g_{2}\right), \\
\vdots \\
\left.\left(B_{k} \cap C_{1}, h_{1} g_{k}\right), \ldots,\left(B_{k} \cap C_{\ell}, h_{\ell} g_{k}\right)\right)
\end{gathered}
$$

where empty intersections are omitted. This gives $\mathcal{F}_{n}^{G}$ the structure of a semigroup (with 
identity element $\left.\left(\left([n], 1_{G}\right)\right)\right)$ satisfying Formulas (1). If $|G|=1$ then $\mathcal{F}_{n}^{G}$ is isomorphic to the face semigroup of the braid arrangement. See [9] for details.

\subsection{The invariant subalgebra}

The action of the symmetric group $S_{n}$ on $[n]$ induces an action on $\mathcal{F}_{n}^{G}$. For example, $\pi \cdot\left(\left(\{1,3\}, g_{1}\right),\left(\{2\}, g_{2}\right)\right)=\left(\left(\{\pi(1), \pi(3)\}, g_{1}\right),\left(\{\pi(2)\}, g_{2}\right)\right)$ for any $\pi \in S_{3}$. This action extends linearly to the semigroup algebra $\mathbb{Z} \mathcal{F}_{n}^{G}$. Consider the subalgebra of invariants under the action of $S_{n}$ :

$$
\left(\mathbb{Z} \mathcal{F}_{n}^{G}\right)^{S_{n}}=\left\{P \in \mathbb{Z} \mathcal{F}_{n}^{G} \mid \pi \cdot P=P \text { for all } \pi \in S_{n}\right\}
$$

That $\left(\mathbb{Z} \mathcal{F}_{n}^{G}\right)^{S_{n}}$ is a subalgebra of $\mathbb{Z} \mathcal{F}_{n}^{G}$ is a consequence of the observation that $\pi \cdot(P Q)=$ $(\pi \cdot P)(\pi \cdot Q)$ for all $\pi \in S_{n}$ and $P, Q \in \mathcal{F}_{n}^{G}$.

As a $\mathbb{Z}$-module $\left(\mathbb{Z} \mathcal{F}_{n}^{G}\right)^{S_{n}}$ is free with a basis indexed by $G$-compositions. By a $G$ composition of $n$ we mean a sequence $\alpha=\left(\left(a_{1}, g_{1}\right), \ldots,\left(a_{k}, g_{k}\right)\right)$ such that $\left(a_{1}, \ldots, a_{k}\right)$ is a composition of $n$, i.e. a list of positive integers summing to $n$, and $g_{i} \in G$ for all $i \in[k]$. In this case we write $\alpha \vDash_{G} n$ and $\ell(\alpha)=k$. The type of an ordered $G$-partition is the $G$-composition defined by

$$
\operatorname{Type}\left(\left(\left(B_{1}, g_{1}\right), \ldots,\left(B_{k}, g_{k}\right)\right)\right)=\left(\left(\left|B_{1}\right|, g_{1}\right), \ldots,\left(\left|B_{k}\right|, g_{k}\right)\right) .
$$

For $\alpha \vDash_{G} n$, let

$$
\sigma_{\alpha}=\sum_{P \in \mathcal{F}_{n}^{G}: \operatorname{Type}(P)=\alpha} P .
$$

Clearly $\left(\sigma_{\alpha}\right)_{\alpha \models_{G} n}$ is a basis for $\left(\mathbb{Z} \mathcal{F}_{n}^{G}\right)^{S_{n}}$.

\subsection{Multiplication rule for the invariant subalgebra}

For $\alpha, \beta, \gamma \vDash_{G} n$, the coefficient of $\sigma_{\gamma}$ in the product $\sigma_{\alpha} \sigma_{\beta}$ is just the number of ways of writing an arbitrary $R \in \mathcal{F}_{n}^{G}$ of type $\gamma$ as a product $R=P Q$ where $\operatorname{Type}(P)=\alpha$ and Type $(Q)=\beta$. Thus, by the multiplication rule for ordered $G$-partitions, we obtain the following multiplication rule inside $\left(\mathbb{Z} \mathcal{F}_{n}^{G}\right)^{S_{n}}$. Consider all $k \times l$ matrices $M$ whose entries are of the form $M_{i j}=0$ or $M_{i j}=(a, g)$ where $a$ is a positive integer and $g \in G$. Let $|0|=0$ and $|(a, g)|=a$, and call $g$ the color of $(a, g)$. Say that $M$ is compatible with $\alpha$ and $\beta$, where $\alpha=\left(\left(a_{1}, g_{1}\right), \ldots,\left(a_{k}, g_{k}\right)\right) \vDash_{G} n$ and $\beta=\left(\left(b_{1}, h_{1}\right), \ldots,\left(b_{\ell}, h_{\ell}\right)\right) \vDash_{G} n$, if the following conditions are satisfied:

(a) For all $i \in[k], \sum_{j=1}^{\ell}\left|M_{i j}\right|=a_{i}$,

(b) For all $j \in[\ell], \sum_{i=1}^{k}\left|M_{i j}\right|=b_{j}$,

(c) For all $i \in[k]$ and $j \in[\ell]$, if $M_{i j} \neq 0$ then $M_{i j}$ has color $h_{j} g_{i}$. 
For a compatible matrix $M$, let $M^{\prime}$ denote the $G$-composition obtained by reading the entries of $M$ row-by-row, omitting entries that are 0 . For example, the following matrix is compatible with $\alpha=\left(\left(4, g_{1}\right),\left(6, g_{2}\right)\right)$ and $\beta=\left(\left(3, h_{1}\right),\left(5, h_{2}\right),\left(2, h_{3}\right)\right)$ :

$$
M=\left(\begin{array}{ccc}
\left(2, h_{1} g_{1}\right) & 0 & \left(2, h_{3} g_{1}\right) \\
\left(1, h_{1} g_{2}\right) & \left(5, h_{2} g_{2}\right) & 0
\end{array}\right)
$$

Here, $M^{\prime}=\left(\left(2, h_{1} g_{1}\right),\left(2, h_{3} g_{1}\right),\left(1, h_{1} g_{2}\right),\left(5, h_{2} g_{2}\right)\right)$.

Proposition 2. Given G-compositions $\alpha=\left(\left(a_{1}, g_{1}\right), \ldots,\left(a_{k}, g_{k}\right)\right)$ and $\beta=\left(\left(b_{1}, h_{1}\right), \ldots\right.$, $\left.\left(b_{\ell}, h_{\ell}\right)\right)$ of $n$, we have

$$
\sigma_{\alpha} \sigma_{\beta}=\sum_{M} \sigma_{M^{\prime}}
$$

where the sum is over all matrices compatible with $\alpha$ and $\beta$.

When $G$ is abelian, Proposition 2 is equivalent to the formula for multiplication inside the generalized descent algebra obtained by Mantaci and Reutenauer [18, Corollary 6.8]. This formula is originally due to Garsia and Remmel [11] for the descent algebra of $S_{n}$.

\subsection{The $G$-descent algebra}

Consider the right permutation action of $S_{n}$ on $G^{[n]}$, the group of functions from $[n]$ to $G$ with multiplication given by $(g h)(i)=g(i) h(i)$ for $g, h \in G^{[n]}$ and $i \in[n]$. A permutation $\pi \in S_{n}$ takes $g \in G^{[n]}$ to $g \cdot \pi$, where $(g \cdot \pi)(i)=g(\pi(i))$. Using this action we construct the wreath product $G<S_{n}$. As a set, $G<S_{n}=S_{n} \times G^{[n]}$. Its group operation is given by $(\pi, g) *(\tau, h)=(\pi \tau,(g \cdot \tau) h)$. It will be convenient to represent an element $(\pi, g) \in G \curlywedge S_{n}$ by $\left(\left(\pi_{1}, g_{1}\right), \ldots,\left(\pi_{n}, g_{n}\right)\right)$, where $\pi_{i}=\pi(i)$ and $g_{i}=g(i)$ for $i \in[n]$. With this notation,

$$
\left(\left(\pi_{1}, g_{1}\right), \ldots,\left(\pi_{n}, g_{n}\right)\right) *\left(\left(\tau_{1}, h_{1}\right), \ldots,\left(\tau_{n}, h_{n}\right)\right)=\left(\left(\pi_{\tau_{1}}, g_{\tau_{1}} h_{1}\right), \ldots,\left(\pi_{\tau_{n}}, g_{\tau_{n}} h_{n}\right)\right) .
$$

This description of $G$ ? $S_{n}$ is consistent with [18].

Given $u=\left(\left(\pi_{1}, g_{1}\right), \ldots,\left(\pi_{n}, g_{n}\right)\right) \in G$ ? $S_{n}$, let $\operatorname{Co}(u)$ denote the unique $G$-composition $\left(\left(a_{1}, h_{1}\right), \ldots,\left(a_{k}, h_{k}\right)\right)$ such that

$$
\begin{array}{ll}
\pi_{1}<\pi_{2}<\cdots<\pi_{a_{1}}, & g_{1}=\cdots=g_{a_{1}}=h_{1}, \\
\pi_{a_{1}+1}<\cdots<\pi_{a_{1}+a_{2}}, & g_{a_{1}+1}=\cdots=g_{a_{1}+a_{2}}=h_{2}, \\
\vdots & \\
\pi_{a_{1}+\cdots+a_{k-1}+1}<\cdots<\pi_{n}, & g_{a_{1}+\cdots+a_{k-1}+1}=\cdots=g_{n}=h_{k},
\end{array}
$$

and where $k$ is as small as possible. Thus, $\operatorname{Co}(u)$ keeps track of those values $i$ such that $\pi_{i}>\pi_{i+1}$ or $g_{i} \neq g_{i+1}$. For instance if $g, h$ are distinct elements in $G$, then

$\mathrm{Co}((3, g),(6, g),(4, g),(1, h),(2, h),(5, h),(8, g),(7, g))=((2, g),(1, g),(3, h),(1, g),(1, g))$.

Let $\mathbb{Z}\left[G \prec S_{n}\right]$ denote the group algebra of $G \imath S_{n}$. For $\alpha \vDash_{G} n$, define $Y_{\alpha} \in \mathbb{Z}\left[G \prec S_{n}\right]$ by

$$
Y_{\alpha}=\sum_{u \in G l S_{n}: \operatorname{Co}(u)=\alpha} u
$$


Clearly the set $\left\{Y_{\alpha} \mid \alpha \vDash_{G} n\right\}$ is linearly independent. Let

$$
\mathcal{D}\left(G \succ S_{n}\right)=\mathbb{Z} \text {-linear span of }\left\{Y_{\alpha} \mid \alpha \vDash_{G} n\right\} \text {. }
$$

The following result is due to Mantaci and Reutenauer [18, Theorem 6.9]:

Theorem 3. If $G$ is abelian then $\mathcal{D}\left(G<S_{n}\right)$ is a subalgebra of $\mathbb{Z}\left[G<S_{n}\right]$.

A generalization of this theorem to arbitrary groups $G$ is discussed in $[4,20]$. We will deduce this more general result from our main theorem. First we will need to introduce another basis for $\mathcal{D}\left(G<S_{n}\right)$. Consider the partial order on the set of $G$-compositions of $n$ generated by cover relations of the form

$$
\left(\left(a_{1}, g_{1}\right), \ldots,\left(a+b, g_{i}\right), \ldots,\left(a_{k}, g_{k}\right)\right)<\left(\left(a_{1}, g_{1}\right), \ldots,\left(a, g_{i}\right),\left(b, g_{i}\right), \ldots,\left(a_{k}, g_{k}\right)\right) .
$$

In other words $\alpha \leq \beta$ if and only if $\beta$ is a color-preserving refinement of $\alpha$. For $\alpha \vDash_{G} n$, let

$$
X_{\alpha}=\sum_{\beta \models_{G} n: \beta \leq \alpha} Y_{\beta}
$$

By Möbius inversion,

$$
Y_{\alpha}=\sum_{\beta \models_{G} n: \beta \leq \alpha}(-1)^{\ell(\alpha)-\ell(\beta)} X_{\beta} .
$$

Thus $\left(X_{\alpha}\right)_{\alpha \models_{G} n}$ is a basis of $\mathcal{D}\left(G<S_{n}\right)$. This basis was introduced in [18] (for $G$ abelian) and has subsequently been used in [4] and [20, 21].

\section{Proof of main result}

We restate and prove the main result announced in the Introduction.

Theorem 1. The $\mathbb{Z}$-module map $f:\left(\mathbb{Z}_{n}^{G}\right)^{S_{n}} \rightarrow \mathbb{Z}\left[G<S_{n}\right]$ defined by $f\left(\sigma_{\alpha}\right)=X_{\alpha}$ is an injective anti-homomorphism of algebras.

Proof. Let $\mathcal{C}$ be the set of ordered $G$-partitions of $[n]$ whose blocks are singletons. Note that $\mathcal{C}$ is a left ideal of $\mathcal{F}_{n}^{G}$. To elaborate, given $P=\left(\left(B_{1}, h_{1}\right),\left(B_{2}, h_{2}\right), \ldots,\left(B_{k}, h_{k}\right)\right) \in \mathcal{F}_{n}^{G}$ and $Q=\left(\left(\left\{\pi_{1}\right\}, g_{1}\right),\left(\left\{\pi_{2}\right\}, g_{2}\right), \ldots,\left(\left\{\pi_{n}\right\}, g_{n}\right)\right) \in \mathcal{C}$, let $\tau \in S_{n}$ be the unique permutation such that

$$
\begin{array}{ll}
B_{1}=\left\{\pi_{\tau_{1}}, \pi_{\tau_{2}}, \ldots, \pi_{\tau_{a_{1}}}\right\}, & \tau_{1}<\cdots<\tau_{a_{1}}, \\
B_{2}=\left\{\pi_{\tau_{a_{1}+1}}, \ldots, \pi_{\tau_{a_{1}+a_{2}}}\right\}, & \tau_{a_{1}+1}<\cdots<\tau_{a_{1}+a_{2}}, \\
\vdots & \\
B_{k}=\left\{\pi_{\tau_{a_{1}+\cdots+a_{k-1}+1}}, \ldots, \pi_{\tau_{n}}\right\}, & \tau_{a_{1}+\cdots+a_{k-1}+1}<\cdots<\tau_{n},
\end{array}
$$


where $a_{i}=\left|B_{i}\right|$ for $i \in[k]$. Then it follows from the definition of multiplication in $\mathcal{F}_{n}^{G}$ that

$$
\begin{aligned}
& P Q=\left(\left(\left\{\pi_{\tau_{1}}\right\}, g_{\tau_{1}} h_{1}\right),\left(\left\{\pi_{\tau_{2}}\right\}, g_{\tau_{2}} h_{1}\right), \ldots,\left(\left\{\pi_{\tau_{a_{1}}}\right\}, g_{\tau_{a_{1}}} h_{1}\right),\right. \\
&\left(\left\{\pi_{\tau_{a_{1}+1}}\right\}, g_{\tau_{a_{1}+1}} h_{2}\right), \ldots,\left(\left\{\pi_{\tau_{a_{1}+a_{2}}}\right\}, g_{\tau_{a_{1}+a_{2}}} h_{2}\right), \\
&\left.\ldots,\left(\left\{\pi_{\tau_{a_{1}+\cdots+a_{k-1}+1}}\right\}, g_{\tau_{a_{1}+\cdots+a_{k-1}+1}} h_{k}\right), \ldots,\left(\left\{\pi_{\tau_{n}}\right\}, g_{\tau_{n}} h_{k}\right)\right) .
\end{aligned}
$$

Consider the action of $\left(\mathbb{Z} \mathcal{F}_{n}^{G}\right)^{S_{n}}$ on the $\mathbb{Z}$-module $\mathbb{Z C}$ by left multiplication. For any $\alpha=\left(\left(a_{1}, h_{1}\right), \ldots,\left(a_{\ell}, h_{k}\right)\right) \vDash_{G} n$ and $\left(\left(\left\{\pi_{1}\right\}, g_{1}\right), \ldots,\left(\left\{\pi_{n}\right\}, g_{n}\right)\right) \in \mathcal{C}$, by $(3)$ we have

$$
\sigma_{\alpha}\left(\left(\left\{\pi_{1}\right\}, g_{1}\right), \ldots,\left(\left\{\pi_{n}\right\}, g_{n}\right)\right)=\sum\left(\left(\left\{\pi_{\tau_{1}}\right\}, g_{\tau_{1}} i_{1}\right), \ldots,\left(\left\{\pi_{\tau_{n}}\right\}, g_{\tau_{n}} i_{n}\right)\right)
$$

where the sum is over all $u=\left(\left(\tau_{1}, i_{1}\right), \ldots,\left(\tau_{n}, i_{n}\right)\right) \in G 2 S_{n}$ such that $\tau_{1}<\cdots<\tau_{a_{1}}$, $\tau_{a_{1}+1}<\cdots<\tau_{a_{1}+a_{2}}, \ldots, \tau_{a_{1}+\cdots+a_{k-1}+1}<\cdots<\tau_{n}$, and $i_{1}=\cdots=i_{a_{1}}=h_{1}, i_{a_{1}+1}=$ $\cdots=i_{a_{1}+a_{2}}=h_{2}, \ldots, i_{a_{1}+\cdots+a_{k-1}+1}=\cdots=i_{n}=h_{k}$. These conditions are equivalent to $\operatorname{Co}(u) \leq \alpha$.

Now identify $\mathcal{C}$ with the set $G \prec S_{n}$ so that if $v=\left(\left(\pi_{1}, g_{1}\right), \ldots,\left(\pi_{n}, g_{n}\right)\right) \in G \prec S_{n}$ then $v$ gets identified with $\left(\left(\left\{\pi_{1}\right\}, g_{1}\right), \ldots,\left(\left\{\pi_{n}\right\}, g_{n}\right)\right) \in \mathcal{C}$. Let $I=\left(\left(1,1_{G}\right),\left(2,1_{G}\right), \ldots,\left(n, 1_{G}\right)\right)$, the identity element of $G \prec S_{n}$. Comparing (4) with (2), we get

$$
\sigma_{\alpha} v=\sum_{u \in G\left(S_{n}: \operatorname{Co}(u) \leq \alpha\right.} v * u=v *\left(\sigma_{\alpha} I\right)
$$

for any $v \in G\left(S_{n}\right.$. In particular, $\sigma_{\alpha} I=X_{\alpha}$.

The map $f$ satisfies $f\left(\sigma_{\alpha}\right)=\sigma_{\alpha} I=X_{\alpha}$, and so $f\left(\sigma_{\alpha} \sigma_{\beta}\right)=\sigma_{\alpha}\left(\sigma_{\beta} I\right)=\left(\sigma_{\beta} I\right) *\left(\sigma_{\alpha} I\right)=$ $X_{\beta} * X_{\alpha}$, completing the proof.

Since the image of $f$ is $\mathcal{D}\left(G<S_{n}\right)$, we have the following corollary:

Corollary 4. For any group $G, \mathcal{D}\left(G 2 S_{n}\right)$ is a subalgebra of $\mathbb{Z}\left[G\left(S_{n}\right]\right.$ and is anti-isomorphic to $\left(\mathbb{Z} \mathcal{F}_{n}^{G}\right)^{S_{n}}$.

\section{Acknowledgments}

I would like to thank the following people for their contributions to this paper. Franco Saliola gave invaluable comments on earlier versions of this manuscript and suggested that I allow $G$ to be non-abelian. Ken Brown shared his insights on how to think about face semigroups combinatorially. Jean-Yves Thibon brought the paper [20] to my attention and provided very instructive comments. Ben Steinberg and Stuart Margolis introduced me to the papers $[19,28,29]$ and clarified their context and relevance, and they gave me generous help on semigroups. The anonymous referee gave valuable suggestions for improving the exposition. 


\section{References}

[1] M. Aguiar and S. Mahajan, Coxeter groups and Hopf algebras. With a foreword by Nantel Bergeron. Fields Institute Monographs, 23. American Mathematical Society, Providence, RI, 2006. xvi+181 pp.

[2] M. Aguiar, N. Bergeron, and K. Nyman, The peak algebra and the descent algebras of types B and D, Trans. Amer. Math. Soc. 356 (2004), 2781-2824.

[3] M. Aguiar, J-C Novelli, and J-Y Thibon, Unital versions of the higher order peak algebras, arXiv:0810.4634.

[4] P. Baumann and C. Hohlweg, A Solomon descent theory for the wreath products G々 $S_{n}$, Trans. Amer. Math. Soc. 360 (2008), 1475-1538.

[5] D. Bayer and P. Diaconis, Trailing the dovetail shuffle to its lair, Ann. Appl. Probab. 2 (1992), no. 2, 294-313.

[6] N. Bergeron and C. Hohlweg, Coloured peak algebras and Hopf algebras, J. Alg. Combin. 24 (2006), no. 3, 299-330.

[7] T.P. Bidigare, Hyperplane arrangements, face algebras, and their associated Markov chains, Ph.D. thesis. University of Michigan, 1997.

[8] C. Bonnafé and C. Hohlweg, Generalized descent algebra and construction of irreducible characters of hyperoctahedral groups, Annales de l'institut Fourier, $\mathbf{5 6}$ (2006), no. 1, 131-181.

[9] K.S. Brown, Semigroups, Rings, and Markov Chains, J. Theoret. Prob. 13 (2000), no. $3,871-938$.

[10] K.S. Brown, Semigroup and ring theoretical methods in probability, Representations of finite dimensional algebras and related topics in Lie theory and geometry, 3-26, Fields Inst. Commun., 40, Amer. Math. Soc., Providence, RI, 2004.

[11] A.M Garsia and J. Remmel, Shuffles of permutations and the Kronecker product, Graphs Combin. 1 (1985), 217-263.

[12] A.M. Garsia and C. Reutenauer, A decomposition of Solomon's Descent Algebra, Adv. Math. 77 (1989), no. 2, 189-262.

[13] I.M. Gessel, Multipartite $P$-partitions and inner products of skew Schur functions, Contemp. Math. 34 (1984), 289-301.

[14] P. Hersh and S.K. Hsiao, Random walks on quasisymmetric functions, arXiv:0709.1477,

[15] C. Hohlweg, Generalized descent algebras, Canad. Math. Bull. 50 (2007), no. 4, $535-546$.

[16] S.K. Hsiao and T.K. Petersen, Colored posets and colored quasisymmetric functions. Ann. Combin, to appear.

[17] C. Malvenuto and C. Reutenauer, Duality between quasi-symmetric functions and the Solomon descent algebra, J. Alg. 177 (1995), no. 3, 967-982. 
[18] R. Mantaci and C. Reutenauer, A generalization of Solomons algebra for hyperoctahedral groups and other wreath products, Comm. Algebra 23 (1995), no. 1, 27-56.

[19] S. Margolis and B. Steinberg, The quiver of an algebra associated to the MantaciReutenauer descent algebra and the homology of regular semigroups, Algebr. Represent. Theory, to appear.

[20] J.-C. Novelli and J.-Y. Thibon, Free quasi-symmetric functions of arbitrary level, arXiv:math/0405597v2

[21] J.-C. Novelli and J.-Y. Thibon, Free quasi-symmetric functions and descent algebras for wreath products, and noncommutative multi-symmetric functions, arXiv:0806.3682

[22] K. Nyman, The peak algebra of the symmetric group, J. Alg. Combin. 17 (2003), 309-322.

[23] M. Petrich and N. R. Reilly, Completely Regular Semigroups, Canad. Math. Soc. Series of Monographs and Advanced Texts 23, Wiley, New York, 1999.

[24] S. Poirier, Cycle type and descent set in wreath products, Discrete Math. 180 (1998), 315-343.

[25] F.V. Saliola, The face semigroup algebra of a hyperplane arrangement, Canadian J. Math., in press.

[26] F.V. Saliola, The quiver of the semigroup algebra of a left regular band, International Journal of Algebra and Computation, 17, no. 8 (2007), 1593-1610.

[27] L. Solomon, A Mackey formula in the group ring of a Coxeter group, J. Alg. 41 (1976), 255-268.

[28] B. Steinberg, Möbius functions and semigroup representation theory, J. Combin. Theory Ser. A 113 (2006), 866-881.

[29] B. Steinberg, Möbius functions and semigroup representation theory II: Character formulas and multiplicities, Adv. Math. 217 (2008), 1521-1557.

[30] J. Tits, Two properties of Coxeter complexes. Appendix to "A Mackey formula in the group ring of a Coxeter group" (J. Algebra 41 (1976), no. 2, 255-264) by Louis Solomon. J. Algebra 41 (1976), no. 2, 265-268.

[31] J. Tits, Buildings of spherical type and finite BN-pairs. Lecture Notes in Mathematics, Vol. 386. Springer-Verlag, Berlin-New York, 1974. x+299 pp. 